\title{
Numerical Solution of the Seventh Order Boundary Value Problems Using B-Spline Method
}

\author{
Maryam Khazaei1, Yeganeh Karamipour² \\ ${ }^{1}$ Department of Computer Science, University of California, Merced, USA \\ ${ }^{2}$ Department of Applied Mathematics, Iran University of Science and Technology, Tehran, Iran \\ Email: mkhazaeipool@ucmerced.edu
}

How to cite this paper: Khazaei, M. and Karamipour, Y. (2021) Numerical Solution of the Seventh Order Boundary Value Problems Using B-Spline Method. Journal of Applied Mathematics and Physics, 9, 3058-3066.

https://doi.org/10.4236/jamp.2021.912198

Received: October 25, 2021

Accepted: December 14, 2021

Published: December 17, 2021

Copyright $\odot 2021$ by author(s) and Scientific Research Publishing Inc. This work is licensed under the Creative Commons Attribution International License (CC BY 4.0).

http://creativecommons.org/licenses/by/4.0/

\begin{abstract}
We develop a numerical method for solving the boundary value problem of The Linear Seventh Ordinary Boundary Value Problem by using the seventh-degree B-Spline function. Formulation is based on particular terms of order of seventh order boundary value problem. We obtain Septic B-Spline formulation and the Collocation B-spline method is formulated as an approximation solution. We apply the presented method to solve an example of seventh order boundary value problem in which the result shows that there is an agreement between approximate solutions and exact solutions. Resulting in low absolute errors shows that the presented numerical method is effective for solving high order boundary value problems. Finally, a general conclusion has been included.
\end{abstract}

\section{Keywords}

Spline and B-Spline Functions, Seventh Order Boundary Value Problem, Septic B-Spline Collocation Method

\section{Introduction}

We consider the linear seventh order differential equation

$$
y^{7}(x)=g(x) y(x)+q(x)
$$

with the boundary conditions

$$
\begin{aligned}
& y(a)=k_{1}, \quad y^{\prime}(a)=k_{2}, \quad y^{\prime \prime}(a)=k_{3}, \quad y^{\prime \prime \prime}(a)=k_{4}, \\
& y(b)=k_{5}, \quad y^{\prime}(b)=k_{6}, \quad y^{\prime \prime}(b)=k_{7} \text {. }
\end{aligned}
$$


Obtaining the analytical solution to this problem is difficult in general. Scientists have used spline functions more commonly due to their useful application in applied mathematics and engineering. Many fields including Computational Mathematics, Mathematical Physics, and Mechanics have played an important role in studying the application of B-Spline [1] methods.

Higher order differential equations have been solved by many numerical and semi-analytical methods. The solutions of differential equations based on spline functions contain high accuracy. There is a comprehensive study with scientists to discuss the numerical solution of linear and nonlinear boundary value problems. For instance, quadratic, cubic, quartic, quintic, sextic, septic and higher degree spline techniques have been used for this purpose. Srivastava [2] has given survey research on cubic, quintic and sextic polynomial and nonpolynomial spline techniques for solving boundary value problems. Geyikli and Gazi Karakoc applied septic B-spline collocation method for the numerical solution of the modified equal width wave equation [3]. A ninth-degree spline function, as well as an eighth degree spline to solve a seventh order boundary value problem, is applied by Parcha Kalyani, Mihretu Nigatu Lemma [4]. The authors obtained an approximate solution matching closely with an exact solution. Fletcher [5] presented the analytic solution of a two dimensional coupled Burgers' equation using the Hopf-Cole transformation. For the various forms of nonlinear PDE's, as model problems in fluid dynamical systems, a variety of studies have been developed [6] [7]. New Cubic B-Spline Approximation for Solving Linear Two-Point Boundary-Value Problems is presented by Busyra Latif, and Samsul Ariffin Abdul Karim [8]. This method is based on cubic B-spline basis functions with a new approximation for the second order derivative. Considering the accuracy of the second-order new approximation by $O(h)^{3}$, the proposed method is $O(h)^{5}$ accurate. Nazir T used a new CBS approximation T for the numerical solutions of coupled viscous Burgers equations as well as a new quintic B-spline approximation for numerical treatment of Boussinesq equation [9] [10]. A new CBS approximation to approximate the solutions to nonlinear third order Korteweg-de Vries equations is used by authors Abbas M and Kashif Iqbal [11].

For finding the numerical solution of a particular case of seventh order linear boundary value problems where some terms of the boundary value problem are zero, we use B-Spline Basis and collocation method which is considered as approximation solution, considering a generic case with a general form of Boundary value problem including term of B-spline basis functions with higher derivatives in the general form of the boundary value problem results in a dense linear system that takes more computational costs with each added term. Thus, resulting in a sparse and banded matrix in the presented method has influenced to have an efficient method in terms of time and cost. Compared with some higher order B-spline techniques, the presented method shows efficient numerical results with fewer absolute errors. The method could also be extended to 
solve higher order boundary value problems.

This paper is organized as follows: In Sections 2 and 3, an introduction and the fundamental definitions of spline, B-spline functions and obtained B-spline basis functions are presented. In Section 3, we consider the collocation method based on B-spline for the linear seventh order boundary value problem. In Section 4, we have discussed the numerical results. In Section 5, the conclusion and further developments are given.

\section{Definitions}

\subsection{Spline}

A spline is described as a piecewise polynomial function that is being as smooth as it can be without reducing it to a polynomial [1]. In general, Spline is a piecewise polynomial function defined in the interval $[a, b]$, such that there exists a decomposition of $D$ into sub-regions in each of which the function is a polynomial of some degree $k$. The term "spline" is used to refer to a wide class of functions that are used in applications requiring data interpolation or smoothing. A function $S(x)$ is a spline [12] of degree $k$ on $[a, b]$ if

$$
\begin{gathered}
S \in C^{k-1}[a, b] \\
a=t_{0}<t_{1}<\cdots<t_{n}=b
\end{gathered}
$$

and

$$
S(x)= \begin{cases}S_{0}(x), & t_{0}<x<t_{1}, \\ S_{1}(x), & t_{1}<x<t_{2}, \\ S_{n-1}(x), & t_{n-1}<x<t_{n}\end{cases}
$$

where

$$
S_{i}(x) \in P^{k}, \quad i=0,1, \cdots, n-1
$$

\subsection{B-Spline}

The B-spline of degree $k$ is denoted by $\varphi_{i}^{k}(x)$, where $i \in Z$.

We define one more parameter to define B-spline basis functions. The $i$-th B-spline basis function of degree $p$, written as $N_{i, p}(u)$, is defined recursively as follows [13]:

$$
\begin{gathered}
N_{i, p}(u)= \begin{cases}1, & \text { if } u_{i+1}>u \geq u_{i} \\
0, & \text { otherwise }\end{cases} \\
N_{i, p}(u)=\frac{u-u_{i}}{u_{i+p}-u_{i}} N_{i, p-1}(u)+\frac{u_{i+p+1}-u}{u_{i+p+1}-u_{i+1}} N_{i+1, p-1}(u)
\end{gathered}
$$

B-spline, or basis spline, is a Spline function that has minimal support with respect to a given degree, smoothness, and domain partition. Any Spline function of a given degree can be expressed as a linear combination of B-splines of 
that degree. Cardinal B-Splines have knots that are equidistant from each other. Here, we denote the B-spline of degree $k$ by $B_{i}^{k}(x)$, where $i$ is an element in $\mathbb{Z}$ with the following properties:

1) $N_{i, p}(u)$ is a degree $p$ polynomial in $u$.

2) Non-negativity: for all $i, p$ and $u, N_{i, p}(u)$ is non-negative.

3) Local support: $N_{i, p}(u)$ is a nonzero polynomial on $\left[u_{i}, u_{i+p+1}\right)$.

4) At most $p+1$ degree $p$ basis functions are nonzero on any span $\left[u_{i}, u_{i+p+1}\right]$, namely: $N_{i-p, p}(u), N_{i-p+1, p}(u), N_{i-p+2, p}(u), \cdots, N_{i, p}(u)$. This property shows that the following basis functions are nonzero on $\left[u_{i}, u_{i+p+1}\right)$.

$$
N_{i-p, p}(u), N_{i-p+1, p}(u), N_{i-p+2, p}(u), \cdots, N_{i, p}(u)
$$

5) Partition of unity: The sum of all nonzero degree $p$ basis functions on span $\left[u_{i}, u_{i+p+1}\right)$ is 1 which states that the sum of these $p+1$ basis functions is 1 .

\section{Obtaining the Septic B-Spline}

We consider equally-spaced knots of a partition $\pi: a=x_{0}<x_{1}<\cdots<x_{n}$ on $[a, b]$. The alternative approach for deriving the B-splines are more applicable with respect to the recurrence relation for the formulations of B-splines of higher degrees. At first, we recall that the $k$ th forward difference $f\left(x_{0}\right)$ of a given function $f(x)$ at $x_{0}$, which is defined recursively by the following [14] and [15]:

$$
\Delta f\left(x_{0}\right)=\Delta f\left(x_{1}\right)-\Delta f\left(x_{0}\right), \quad \Delta^{k+1} f\left(x_{0}\right)=\Delta^{k} f\left(x_{1}\right)-\Delta^{k} f\left(x_{0}\right)
$$

Definition: The function $(x-t)_{+}^{m}[2]$

$$
(x-t)_{+}^{m}= \begin{cases}(x-t)^{m}, & x \leq t_{0} \\ 0, & x<t\end{cases}
$$

It is clear that $(x-t)_{+}^{m}$ is $(m-1)$ times continuously-differentiable with respect to $t$ and $x$. The B-spline of order $m$ is defined as follows:

$$
B_{i}^{m}(t)=\frac{1}{h^{m}} \sum_{j=0}^{m+1}(-1)^{m+1-j}\left(x_{i-2+j}-t\right)_{+}^{m}=\frac{1}{h^{m}} \Delta^{m+1}\left(x_{i-2}-t\right)_{+}^{m}
$$

Considering various values of $m$. Let $m=1$, turns out the B-spline of various orders.

$$
\begin{gathered}
\frac{1}{h^{1}} \Delta^{2}\left(x_{i-2}-t\right)_{+}^{1}=\frac{1}{h^{1}}\left[\left(x_{i-2}-t\right)_{+}-2\left(x_{i-1}-t\right)_{+}+\left(x_{i}-t\right)_{+}\right] \\
B_{i}^{m}(t)= \begin{cases}\left(x_{i-2}-t\right)-2\left(x_{i-1}-t\right), & x_{i-2}<t \leq x_{i-1} \\
\left(x_{i}-t\right), & x_{i-1}<t \leq x_{i} \\
0, & \text { otherwise }\end{cases}
\end{gathered}
$$

By considering different values for $m$, we can get different degrees of B-Spline. To obtain Septic B-spline basis functions, we need to partition the interval $[a, b]$ while we need to choose mesh points such that $a=x_{0}, \cdots, b=x_{n}$ and $x_{i}=a+i h, i=0,1, \cdots, N$ where $h=(b-a) / n$. 


$$
B_{i}(x)=\frac{1}{h^{7}} \begin{cases}\left(X-X_{j-4}\right)^{7}, & X \in\left[X_{j-4}, X_{j-3}\right] \\ \left(X-X_{j-4}\right)^{7}-8\left(X-X_{j-3}\right)^{7}, & X \in\left[X_{j-3}, X_{j-2}\right] \\ \left(X-X_{j-4}\right)^{7}-8\left(X-X_{j-3}\right)^{7}+28\left(X-X_{j-2}\right)^{7}, & X \in\left[X_{j-2}, X_{j-1}\right] \\ \left(X-X_{j-4}\right)^{7}-8\left(X-X_{j-3}\right)^{7}+28\left(X-X_{j-2}\right)^{7}-56\left(X-X_{j-1}\right)^{7}, & X \in\left[X_{j-1}, X_{j}\right] \\ \left(X_{j+4}-X\right)^{7}-8\left(X_{j+3}-X\right)^{7}+28\left(X_{j+2}-X\right)^{7}-56\left(X_{j+1}-X\right)^{7}+28\left(X_{j+2}-X\right)^{7}, & X \in\left[X_{j}, X_{j+1}\right] \\ \left(X_{j+4}-X\right)^{7}-8\left(X_{j+3}-X\right)^{7}, & X \in\left[X_{j+1}, X_{j+2}\right] \\ \left(X_{j+4}-X\right)^{7}, & X \in\left[X_{j+2}, X_{j+3}\right] \\ 0, & X \in\left[X_{j+2}, X_{j+3}\right]\end{cases}
$$

let $S_{7}[\pi]$ be the space of continuously differentiable, piecewise seventh-degree polynomials on $\pi$. Using [12] [16], the B-splines and its derivatives are defined in the Tables 1-3 respectively. Table 1 with the value of $B_{i}^{\prime \prime \prime}(x)$ and $B_{i}^{4}(x)$, and Table 2 with the value of $B_{i}^{5}(x)$ and $B_{i}^{6}(x)$ and finally, Table 3 The value of $B_{i}^{7}(x)$ and $B_{i}^{6}(x)$ are shown respectively. We also point out that $B_{i}(x)=0$ for $x<x_{i-4}$ and $x>x_{i+4}$.

\section{Collocation Method Based on B-Spline for Linear Seven Order Boundary Value Problem}

The approximate solution of Equation (1.1) by Collocation method based on septic B-spline is as follows

$$
y(x)=\sum_{j=-7}^{n} \alpha_{j} \beta_{j}(x)
$$

Table 1. Values of $B_{i}^{3}(x)$ and $B_{i}^{4}(x)$.

\begin{tabular}{ccccccccc}
\hline$x_{i-4}$ & $x_{i-3}$ & $x_{i-2}$ & $x_{i-1}$ & $x_{i}$ & $x_{i+1}$ & $x_{i+2}$ & $x_{i+3}$ & $x_{i+4}$ \\
\hline 0 & $210 / h^{3}$ & $1680 / h^{3}$ & $-3990 / h^{3}$ & 0 & $3990 / h^{3}$ & $-1680 / h^{3}$ & $-210 / h^{3}$ & 0 \\
0 & $840 / h^{4}$ & 0 & $-7560 / h^{4}$ & $13,440 / h^{4}$ & $-7560 / h^{4}$ & 0 & $840 / h^{4}$ & 0
\end{tabular}

Table 2. Values of $B_{i}^{5}(x)$ and $B_{i}^{6}(x)$.

\begin{tabular}{ccccccccc}
\hline$x_{i-4}$ & $x_{i-3}$ & $x_{i-2}$ & $x_{i-1}$ & $x_{i}$ & $x_{i+1}$ & $x_{i+2}$ & $x_{i+3}$ & $x_{i+4}$ \\
\hline 0 & $2520 / h^{5}$ & $-10,080 / h^{5}$ & $12,600 / h^{5}$ & 0 & $-12,600 / h^{5}$ & $10,080 / h^{5}$ & $-2520 / h^{5}$ & 0 \\
0 & $5040 / h^{6}$ & $-30,240 / h^{6}$ & $75,600 / h^{6}$ & $-100,800 / h^{6}$ & $75,600 / h^{6}$ & $-30,240 / h^{6}$ & $5040 / h^{6}$ & 0 \\
\hline
\end{tabular}

Table 3. Values of $B_{i}^{7}(x)$.

\begin{tabular}{ccccccccc}
\hline$x_{i-4}$ & $x_{i-3}$ & $x_{i-2}$ & $x_{i-1}$ & $x_{i}$ & $x_{i+1}$ & $x_{i+2}$ & $x_{i+3}$ & $x_{i+4}$ \\
\hline 0 & $5040 / h^{7}$ & $-35,280 / h^{7}$ & $105,840 / h^{7}$ & $-176,400 / h^{7}$ & $-176,400 / h^{7}$ & $105,840 / h^{7}$ & $-35,280 / h^{7}$ & 0 \\
\hline
\end{tabular}


where $\alpha_{j}$, are unknown real coefficients to be determine and $B_{j}(x)$ are seven degree B-spline function and let $x_{0}, x_{1}, \cdots, x_{n}$ be $n+1$ grid points in the interval $[a, b]$, so that

$$
x_{i}=a+i h, \quad i=0,1, \cdots, n, \quad x_{0}=a, \quad x_{n}=b, \quad h=(b-a) / n
$$

with substituting (12) in (1), we get the following

$$
\left[\sum_{j=-7}^{n} \alpha_{j}\left(\beta_{j}^{7}\left(x_{i}\right)-g\left(x_{i}\right) \beta_{j}\left(x_{i}\right)\right)-q\left(x_{i}\right)\right]=0, \quad i=0,1, \cdots, n
$$

We use boundary conditions (2) and (12) to make the following system to evaluate the $\alpha_{j}$ so that we get the following:

$$
\begin{gathered}
{\left[\sum_{j=-7}^{n} \alpha_{j}\left(\beta_{j}^{7}\left(x_{i}\right)-g\left(x_{i}\right) \beta_{j}\left(x_{i}\right)\right)-q\left(x_{i}\right)\right]=0, \quad i=0,1, \cdots, n} \\
\sum_{j=-7}^{n} \alpha_{j} \beta_{j}\left(x_{i}\right)=y(a), \quad i=n+1 \\
\sum_{j=-7}^{n} \alpha_{j} \beta_{j}^{\prime}\left(x_{i}\right)=y^{\prime}(a), \quad i=n+2 \\
\sum_{j=-7}^{n} \alpha_{j} \beta_{j}^{\prime \prime}\left(x_{i}\right)=y^{\prime}(a), \quad i=n+3 \\
\sum_{j=-7}^{n} \alpha_{j} \beta_{j}^{\prime \prime \prime}\left(x_{i}\right)=y^{\prime \prime \prime}(a), \quad i=n+4 \\
\sum_{j=-7}^{n} \alpha_{j} \beta_{j}^{\prime \prime}\left(x_{i}\right)=y^{\prime \prime}(b), \quad i=n+5 \\
\sum_{j=-7}^{n} \alpha_{j} \beta_{j}^{\prime}\left(x_{i}\right)=y^{\prime}(b), \quad i=n+6 \\
\sum_{j=-7}^{n} \alpha_{j} \beta_{j}\left(x_{i}\right)=y(b), \quad i=n+7
\end{gathered}
$$

Using Tables 1-3 and (3.4) to (3.11), we evaluate the values of B-Spline functions at the knots $x_{i}, i=0, \cdots, n$ A system $A$ of $n+8$ linear equations in the $(n+8)$ unknowns $\alpha_{-7}, \alpha_{-6}, \cdots, \alpha_{n}$ is obtained. This system can be written in a matrix-vector form as follows

$$
A \alpha=X
$$

where

$$
\alpha=\left[\alpha_{-7}, \alpha_{-6}, \cdots, \alpha_{n}\right]^{\mathrm{T}}
$$

and

$$
\left.X=\left[q\left(x_{0}\right), \cdots, q\left(x_{n}\right), y(a), y^{\prime}(a), y^{\prime \prime}(a), y^{\prime \prime \prime}(a), y^{\prime \prime}(b)\right), y^{\prime}(b), y(b)\right]^{\mathrm{T}}
$$

The matrix $A$ is a linear matrix which is sparse and considered as the banded matrix which is a sparse matrix whose nonzero entries are confined to a diagonal band, comprising the main diagonal and zero or more diagonals on either side. These properties of matrix $A$ leads to more efficiency and less computational cost 
Table 4. The values of the exact solution, approximate solution and max absolute errors for problem 1 .

\begin{tabular}{cccc}
\hline$n$ & $Y_{i}$ & $y_{i}$ & Max absolute Error \\
\hline 20 & 0.35094702 & 0.33068119 & 0.020265825 \\
40 & 0.80970360 & 0.80296795 & 0.00673565 \\
60 & 0.99985955 & 0.9921557165 & 0.00602099 \\
80 & 1.00000000 & 0.99969918 & 0.00030082 \\
\hline
\end{tabular}

for the method as well as the algorithm of Cholesky or the $L D L^{\mathrm{T}}$ factorization can take advantage of the structure banded matrices. By solving the linear system, the values of

$$
\alpha=\left[\alpha_{-7}, \alpha_{-6}, \cdots, \alpha_{n}\right]^{\mathrm{T}}
$$

can be obtained, then the approximate solution of Equation (3.1) will be evaluated by using (3.12).

\section{Numerical Results}

In this section, we solve a linear problem that will be solved by septic B-Spline functions.

Example: First, We consider the linear boundary value problem [17].

$$
y^{7}(x)=y(x)-7 \mathrm{e}^{x}, \quad 0 \leq x \leq 1
$$

with the following boundary conditions

$$
\begin{aligned}
& y(0)=1, \quad y(1)=0, \quad y^{\prime}(1)=\mathrm{e}, \quad y^{\prime \prime}(1)=2 \mathrm{e}, \\
& y^{\prime}(0)=0, \quad y^{\prime \prime}(0)=1, \quad y^{\prime \prime \prime}(0)=2 .
\end{aligned}
$$

The domain $[0,1]$ is divided into 20,40,60,80 equal sub-intervals. we define mesh-points $x_{i}$ as $x_{i}=x_{0}+i h$ where $h$ is $h=\left(b=x_{n}-a=x_{0}\right) / n$. Maximum absolute error and approximate solution $y_{i}$ coming from the presented method and exact solution $Y_{i}$ for this problem are shown in Table 4, which the analytical solution is $(1-x) \mathrm{e}^{x}$. A comparison for the absolute error of [18] and ninth-degree spline approximation for Example 1 at $h=0.1$, turns out that [18] has the larger absolute error rather than our presented method. We could divide the domain into different sizes of mesh points, it will increase the accuracy when $n=100,120,160$, and 180 . Having a sparse banded linear system for the given example turns out less computational time and a more efficient solution.

\section{Conclusion}

For finding the numerical solution of a particular case of seventh order linear boundary value problems where some terms of the BVP are zero, we use B-Spline Basis and Collocation method which is considered as an approximation solution. We evaluate the solutions for problem by the seventh degree B-spline. We end up with a banded sparse matrix with the property of being diagonal that takes less computational cost than some other methods [18] which they consider 
a general form of Boundary value problem. The reason is that there will be more terms of B-spline basis functions with higher derivatives in the general form of the boundary value problem. Then, the linear system form will be contained a more dense matrix which takes more computational cost with each added term. Therefore, the presented method will be required less running time. Numerical results show the capability and efficiency of the present method. Numerical results showed that the method achieved forth order accuracy. One of the importances of this method is that we can approximate the solution at every point of the range of domain. Numerical results obtained by the present method are in good agreement with the exact solutions or numerical solutions available in the literature. We can extend the degree of the proposed method to solve the higher (i.e., more than 7 th) order boundary value problem. The presented method also could be used for the nonlinear form of seventh order Boundary value problems with the same B-Spline basis functions.

\section{Conflicts of Interest}

The authors declare no conflicts of interest regarding the publication of this paper.

\section{References}

[1] Schumaker, L.L. (1981) Spline Functions: Basic Theory. John Wiley and Son, New York.

[2] Srivastava, P.K. (2014) Study of Differential Equations with Their Polynomial and Non-Polynomial Spline Based Approximation. Acta Tehnica Corviniensis-Bulletin of Engineering, 7, 2067-3809.

[3] Geyikli, T. and Gazi Karakoc, S.B. (2011) Septic B-Spline Collocation Method for the Numerical Solution of the Modified Equal Width Wave Equation. Inonu University, Malatya. https://doi.org/10.4236/am.2011.26098

[4] Parcha, K. and Mihretu, N.L. (2016) Solutions of Seventh Order Boundary Value Problems Using Ninth Degree Spline Functions and Comparison with Eighth Degree Spline Solutions. Journal of Applied Mathematics and Physics, 4, 249-261. https://doi.org/10.4236/jamp.2016.42032

[5] Fletcher, C. (1983) Generating Exact Solutions of the Two-Dimensional Burgers Equation. The International Journal for Numerical Methods in Fluids, 203, 213-216. https://doi.org/10.1002/fld.1650030302

[6] Sayed, T.E. and Danaf, A.E. (2002) Numerical Solution of the Korteweg-Vries Burgers Equation by Quintic Spline Method. Studia Universitatis Babes-Bolyai Mathematica, 47, 41-55.

[7] Liu, W. (2002) A Asymptotic Behavior of Solutions of Time-Delayed Burgers Equation. Discrete and Continuous Dynamic Systems, Series B, 2, 47-56. https://doi.org/10.3934/dcdsb.2002.2.47

[8] Latif, B., Abdul Karim, S. and Hashim, I. (2021) New Cubic B-Spline Approximation for Solving Linear Two-Point Boundary-Value Problems. Mathematics, 9, Article No. 1250. https://doi.org/10.3390/math9111250

[9] Nazir, T., Abbas, M. and Iqbal, M.K. (2020) New Cubic B-Spline Approximation Technique for Numerical Solutions of Coupled Viscous Burgers Equations. Engi- 
neering Computations, 38, 83-106. https://doi.org/10.1108/EC-08-2019-0365

[10] Nazir, T., Abbas, M. and Iqbal, M.K. (2019) A New Quintic B-Spline Approximation for Numerical Treatment of Boussinesq Equation. Journal of Mathematics and Computer Science, 20, 30-42. https://doi.org/10.22436/jmcs.020.01.04

[11] Abbas, M., Kashif Iqbal, M., Zafar, B. and Mat Zin, S.B. (2019) New Cubic B-Spline Approximations for Solving Non-Linear Third-Order Korteweg-de Vries Equation. Indian Journal of Science and Technology, 12, 1-9. https://doi.org/10.17485/ijst/2019/v12i6/141953

[12] Rashidinia, J., Khazaei, M. and Nikmarvani, H. (2015) Spline Collocation Method for Solution of Higher Order Linear Boundary Value Problems. TWMS Journal of Pure and Applied Mathematics, 6, 38-47.

[13] Ching-KuangShene, Department of Computer Science Michigan Technological University. https://pages.mtu.edu/ shene/

[14] Rashidinia, J. and Sharifi, Sh. (2011) Survey of B-Spline Functions to Approximate the Solution of Mathematical Problems. Iran University of Science and Technology, Tehran. https://doi.org/10.1186/2251-7456-6-48

[15] Printer, P.M. (1975) Splines and Variational Methods. Colorado State University, Fort Collins, Wiley Classics Edition, Vol. 57, 421.

[16] De Boor, C. (2001) A Practical Guide to Splines. Revised Edition, Springer, New York. (Original Edition 1978) https://doi.org/10.1007/978-1-4612-6333-3

[17] Akram, G., Shahid, S.S. and Iftikhar, I. (2012) Solution of Seventh Order Boundary Value Problems by Variational Iteration Technique. Applied Mathematical Sciences, 6, 4663-4672.

[18] Rajashekhar Reddy, Y. (2017) Efficient Numerical Solution for Seventh Order Differential Equation by Using Septic B-Spline Collocation Method with Non-Uniform Length. Global Journal of Pure and Applied Mathematics, 13, 3543-3548. 